\title{
Copper Through Silicon Vias Studied by Photo-elastic Scanning Infrared Microscopy
}

\author{
M. Herms ${ }^{\mathrm{a}}$, M. Wagner ${ }^{\mathrm{a}}$, I. De Wolf ${ }^{\mathrm{b}, \mathrm{c}}$ \\ ${ }^{a}$ PVA Metrology \& Plasma Solutions GmbH, Am Naßtal 6/8, D-07751 Jena-Maua, Germany \\ ${ }^{b}$ imec, Kapeldreef 75, B-3001 Leuven, Belgium \\ ${ }^{c}$ Dept. Materials Science, Fac. Engineering, KU Leuven, B-3000 Leuven, Belgium
}

Corresponding author: martin.herms@pvatepla.com, Tel: +49 (03641) 61389 851; Fax: +49 (03641) 6138950

\begin{abstract}
The in-plane stress distribution in copper through silicon vias (TSV) ensembles of different design has been studied by the scanning infrared stress explorer (SIREX). SIREX is a reflection-based plane polarimeter particularly developed for the high-resolution stress state visualization in silicon-based electronic and mechanic devices. The SIREX method is based on the principle of stress-induced birefringence. We demonstrate that the silicon crystal matrix around the TSV is optically anisotropic with a stress distribution being similar to fields which are known from point-like stress sources. The maps of optical anisotropy have been converted into maps of difference of principal stress components $\Delta \sigma$ with a resolution of a few $\mathrm{kPa}$. We show that magnitude and direction of $\Delta \sigma$ depend on the geometrical design of the TSVs, in particular on length and diameter. The average radial profile of magnitude is discussed. In consequence, we offer a promising tool and method for the non-destructive evaluation of TSV structures in view of their stress characteristics and integrity.
\end{abstract}

Keywords: copper through silicon vias, scanning infrared stress explorer, SIREX, stress-induced birefringence

\section{Introduction}

For the three-dimensional stacking of electronic chips, TSV (through silicon vias) are one of the key elements. TSV are essential holes in Si filled with metal, e. g. copper, i. e. with a material of higher thermal expansion than silicon. Because of the processing induced residual stress in the Si near a TSV, which can affect the functionality of electronic devices, a keep-out zone has to be defined. On the other hand, the integrity of the metal filling has to be checked, if possible by non-destructive analytical tools.

Recently, we have presented the photo-elastic microscope SIREX (Scanning Infrared Stress Explorer) as promising complementary tool to characterize TSVs in comparison to micro-Raman spectroscopy and Scanning Acoustic Microscopy [1]. SIREX works nondestructively and relatively fast in view of the application to failure inspection under industrial conditions $\left(1 \mathrm{~mm}^{2} \mathrm{~min}^{-1}\right)$. The sensitivity to visualize differences in stress of $\leq 1 \mathrm{kPa}$ is unrivaled.

Generally, the design of a TSV either under development or already applied is versatile. The main features of a single TSV are length and diameter. For a TSV group we have additionally to take into account number, setting and pitch. The standard filling material is copper but alternatives like tungsten are also applied. Different processes of fabrication and treatment are a well-protected know-how. Therefore, the aim of the paper is not to discuss details of TSV fabrication but to demonstrate the potential of SIREX for helping to optimize them.

\section{Method \\ 2.1 SIREX}

The scheme of SIREX is shown in Fig. 1. SIREX 
is a so-called plane polarimeter. This means SIREX uses a linearly polarized probing laser beam and measures the depolarization after penetrating the sample. The depolarization is the ratio of intensities measured by two detectors, perpendicularly and parallel to the initial polarization. SIREX works in reflection, i. e. the probing laser beam is reflected either from the back face or other inner faces of the sample. SIREX uses an infrared laser beam of $1.3 \mu \mathrm{m}$ wavelength. SIREX does not work depth-resolving but the probing beam integrates twice over the whole path between front and reflecting face.

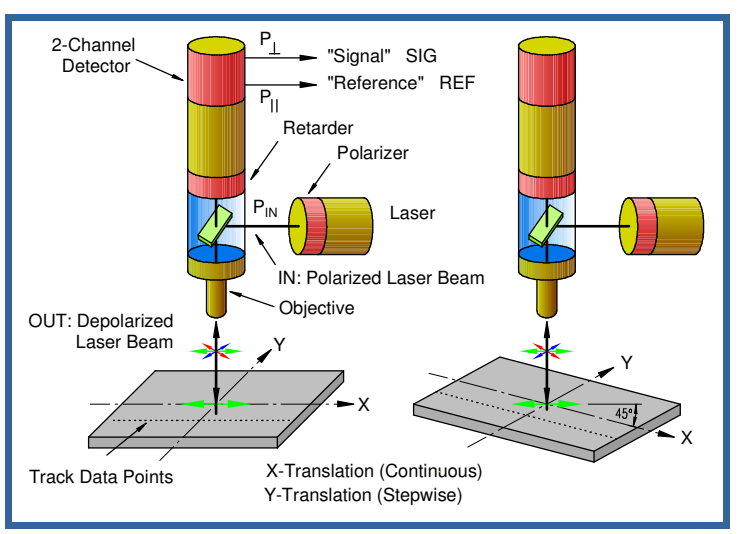

Fig. 01: Scheme of SIREX showing the path of probing beam from the laser source to the sample, after reflection from the backside of sample to the detectors. The initial polarization angle is varied by turning the sample holder (see [1], [6]).

The first basic assumption usually postulated for applications like that here described is that the depolarization is only caused by optical birefringence and not by scattering on structures or roughness of the faces. Optical birefringence appears in optically anisotropic materials. This means that the principal components of the refraction index $\mathrm{n}$ are different. Hence, the in-plane lateral orientation dependency of $n$ can be pictured as ellipses. In consequence, a plane polarimeter detects only depolarized shares of light if the initial polarization is not aligned parallel or perpendicularly to the principal axis of $\mathrm{n}_{1,2}$. Thus, SIREX detects only the shear components resulting in the difference $\Delta \mathrm{n}=\mathrm{n}_{1}-\mathrm{n}_{2}$ (see more details in the next paragraph).

The second basic assumption is that the optical anisotropy is caused by stress $\sigma$. In the case of (100) silicon the native birefringence can be neglected. The relation between $\Delta \mathrm{n}$ and $\Delta \sigma$ is given by the Wertheim law. Because of the "in-plane principle" and the integrating probe, SIREX can not give direct information about normal stress components, in particular about normal stress gradients.

Using a special configuration and signal processing called ARD (alternating retarder depolarization; see [2]) the maps of depolarization are converted into those of the shear stress equivalent $\mathrm{G}$. $\mathrm{G}$ is proportional to the shear stress $\tau=\Delta \sigma \sin (2 \gamma)$ where $\gamma$ is the angle between initial polarization and the fast principal axis $\mathrm{n}_{1}$ and $\sigma_{1}$, respectively. In order to eliminate the dependency of $\Delta \sigma$ on $\gamma$ we use the socalled multi-polarization analysis. This means we calculate a map of $\Delta \sigma$ from a set of single maps of $\tau$ by varying $\gamma$, i. e. by turning the $x-y$ stage (see fig. 01).

\subsection{Shear stress maps of point-like stress sources}

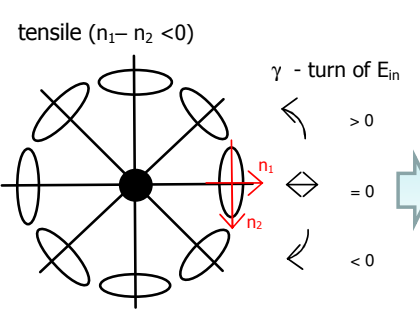

$\mathrm{E}_{\text {in }}<\otimes \rightarrow$ map of $\mathrm{G}$

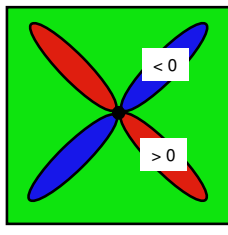

wafer plane

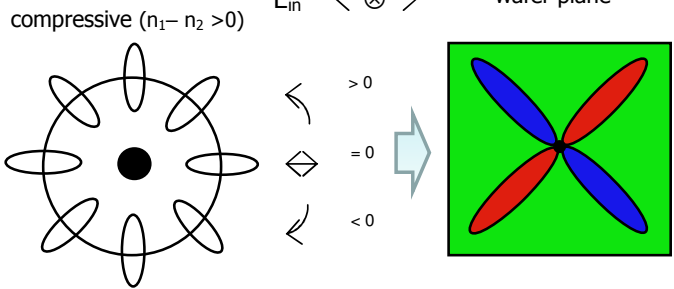

Fig. 02: Ellipses of optical anisotropy surrounding point-like sources of tensile and compressive stress in radius direction (left side) and the shear stress equivalent maps caused by turn of polarization of the probing laser (right side).

"Point-like" stress sources are ideal targets for SIREX provided that the characteristic dimension is not smaller than the lateral resolution of $3 \mu \mathrm{m}$. Polarimeters such as SIREX do profit from the fact that 
the extension of the stress fields is usually one order of magnitude larger than that of the related source itself. Ideal point-like stress sources generate circular stress fields. Consequently, the optical anisotropy is also radial-symmetric if the material is elastically isotropic. The radial decay of the shear stress components of a single dislocation threading the sample in normal direction are described by $\mathrm{a}^{-1}$ law [3]. For pin marks formed during high-temperature processing a $\mathrm{R}^{-2}$ decay seems to be adequate provided that the matrix around the pillar does not show further generation of defects like slip-lines [4]. TSV are holes filled with a material of thermal expansion coefficient being significantly different from silicon. The description of stress decay near TSVs is often done using the Lamé law. There it is assumed that there is no gradient in the normal direction [5].

Fig. 2 demonstrates the change of depolarization of a linearly polarized laser beam by point-like stress sources. The matrix around is either tensile or compressively stressed. Consequently, the fast axis $n_{1}$ is aligned either in or perpendicularly to the radius direction. Depending on the sign of angle at which the laser polarization cut the ellipse the polarization is subsequently turned clockwise or anti-clockwise. Hence, we obtain "butterflies" of G where the colour sequence depends on the stress direction.

\section{Study of TSV \\ 3.1 Experimental}

In this paper we discuss selected designs of TSVs: single TSVs and two groups of 3-fold and 5-fold TSVs, respectively, which are arranged to matrices with different pitches between 90 and $200 \mu \mathrm{m}$. Each single TSV is a blind hole filled with copper. The thin isolation liner between $\mathrm{Cu}$ and $\mathrm{Si}$ in the TSV is neglected in this discussion. The length of the single TSV of the 3-fold group is $50 \mu \mathrm{m}$, that of the 5-fold group $100 \mu \mathrm{m}$. The diameters are 3 and $10 \mu \mathrm{m}$, respectively. The thickness of the wafer pieces is ca. $770 \mu \mathrm{m}$.

Because of former successful experiments on laser marks as well as first time results on TSVs [6] the measurements have been performed in face-down configuration (see Fig. 03). This means the beam is reflected either from the front face or from the TSV bottom. The reflection maps in Fig. 03 demonstrate that the lateral resolution is sufficient to separate the single TSV.
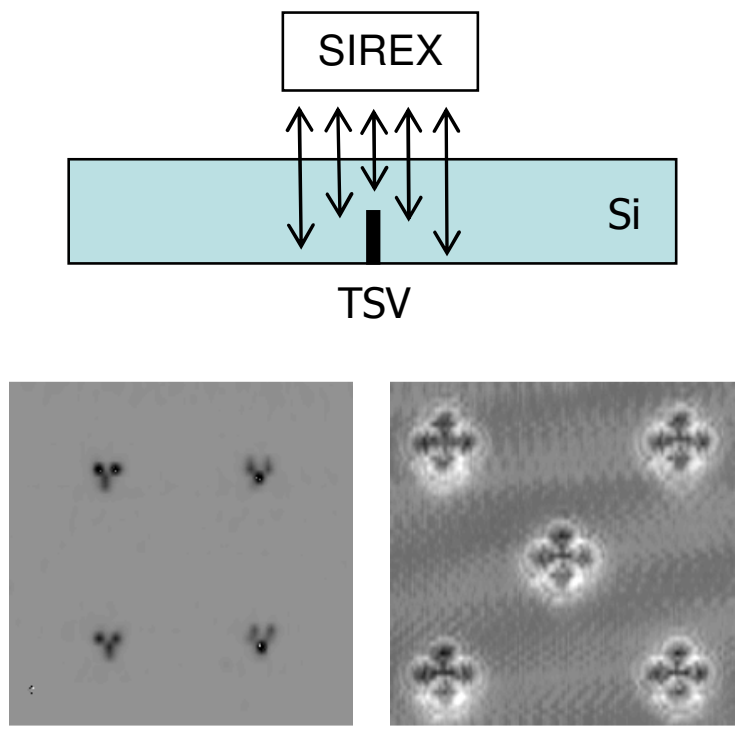

Fig. 03: Scheme of face-down measurement configuration (above) and reflection maps of 3-fold TSVs (left, $210 \mu \mathrm{m} \times 210 \mu \mathrm{m}$ ) and 5-fold TSVs (right, $350 \mu \mathrm{m} \times 350 \mu \mathrm{m}$ ). The diameter of the single TSVs is 3 and $10 \mu \mathrm{m}$, the length 50 and $100 \mu \mathrm{m}$, respectively.

\subsection{Results}

\subsubsection{Single TSV}
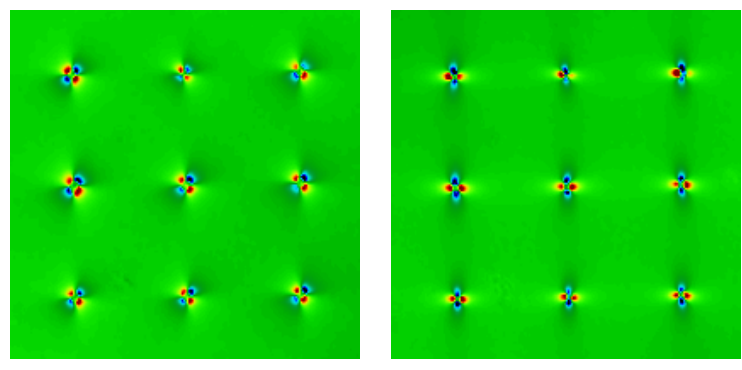

Fig. 04: Shear stress equivalent maps $(625 \mu \mathrm{m} \mathrm{x}$ $625 \mu \mathrm{m}$ ) of $3 \times 3$ matrix of single TSV (length $50 \mu \mathrm{m}$, diameter $3 \mu \mathrm{m}$ ) using $0^{\circ}$ (left) and $45^{\circ}$ (right) polarization

Fig. 04 shows the $G$ maps as recorded on the single TSVs (length $50 \mu \mathrm{m}$, diameter $3 \mu \mathrm{m}$ ) using different initial polarization $\left(0^{\circ}\right.$ and $\left.45^{\circ}\right)$. The butterflies are turning with the initial polarization. The 
colour sequence reveals a tensile stress field (see Fig. 02 in comparison).

Fig. 05 shows the centre TSV as one detail of the calculated $\Delta \sigma$ map. The white lines represent the direction of the "fast" axis of anisotropy $\mathrm{n}_{1}$ and $\sigma_{1}$, respectively. Before, a global field aligned diagonally was eliminated by high-pass filtering. Thus, we get the local TSV field what is radial-symmetric but in the central part slightly four-fold deformed probably caused by the elastic anisotropy of silicon in [100] and [110] direction.

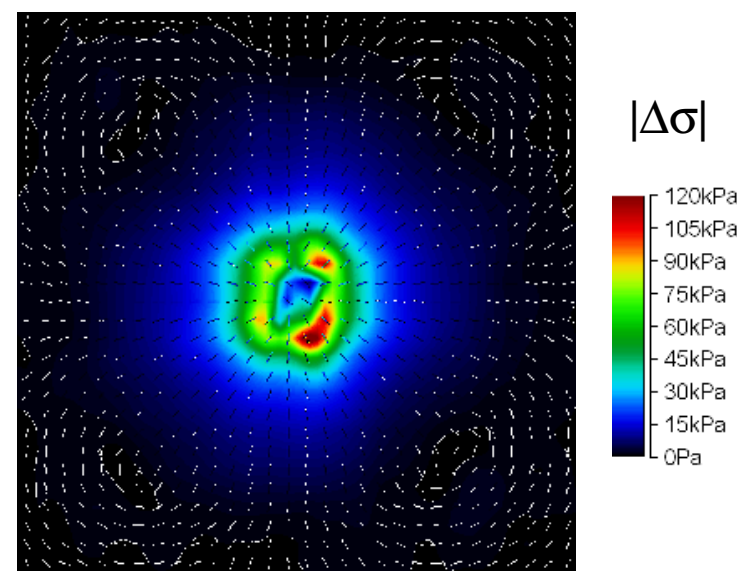

Fig. 05: Calculated $\Delta \sigma$ map $(150 \mu \mathrm{m} \times 150 \mu \mathrm{m})$ of a single TSV

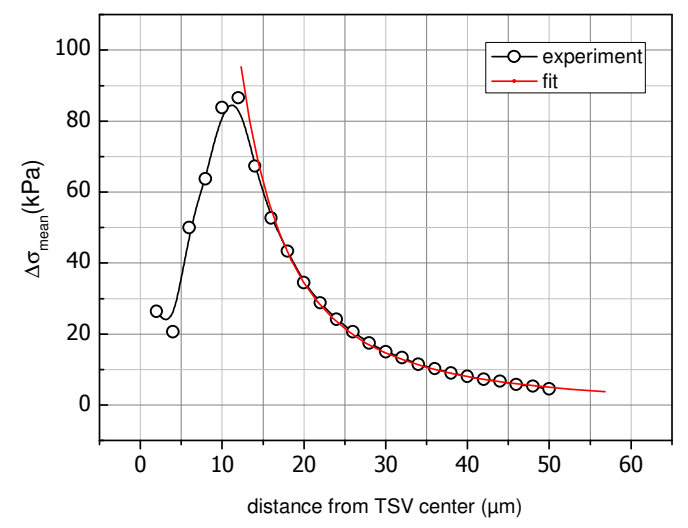

Fig. 06: Radial profile of single TSV after averaging in rings of $2 \mu \mathrm{m}$ width (see Fig. 05) and fit with $\mathrm{f} \sim \mathrm{R}^{-\mathrm{n}}(\mathrm{n}=2.1)$

Fig. 06 presents the radial profile what we have got by averaging $\Delta \sigma$ in rings around the TSV centre. We observe a steep increase for $R \leq 10 R_{T S V}$ but for $R \geq 10$ $\mathrm{R}_{\mathrm{TSV}}$ the decay follows roughly a $\mathrm{R}^{-2}$ law.

\subsubsection{3-fold TSVs group}

Following the same procedure, Fig. 07 shows the G maps of the 3-fold TSV matrix as measured with different initial polarization. The colour sequence reveals tensile stress fields surrounding the TSV groups.
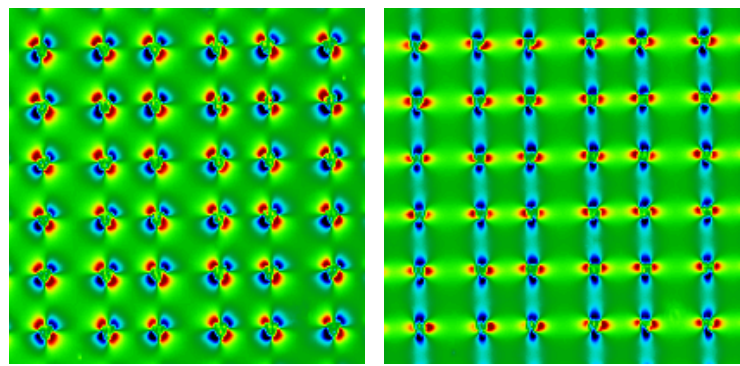

Fig. 07: Shear stress equivalent maps $(625 \mu \mathrm{m} \mathrm{x}$ $625 \mu \mathrm{m}$ ) of $6 \times 6$ matrix of 3-fold TSV (length $50 \mu \mathrm{m}$, diameter $3 \mu \mathrm{m}$ ) using $0^{\circ}$ (left) and $45^{\circ}$ (right) polarization

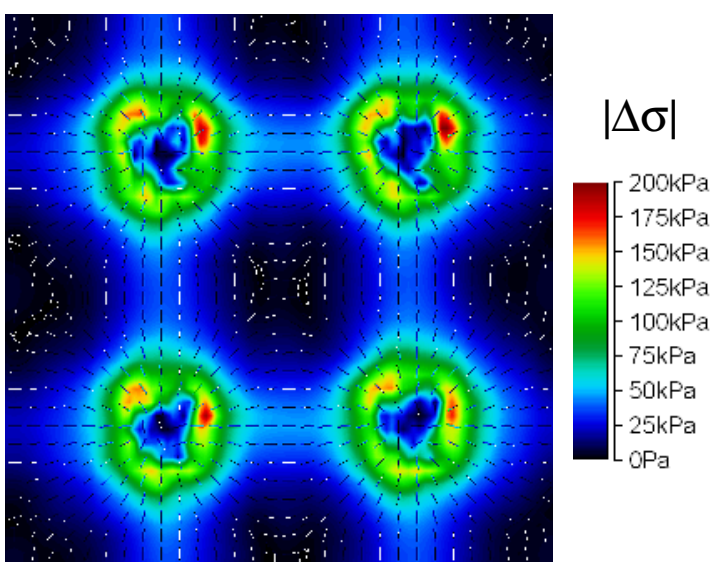

Fig. 08: Calculated $\Delta \sigma$ map $(200 \mu \mathrm{m} \times 200 \mu \mathrm{m})$ of 3fold TSV

Fig. 08 presents the related calculated maps of $\Delta \sigma$. The white lines confirm that the fast axis of anisotropy is aligned in radius direction. Furthermore, we have also determined the radial profile by averaging in rings 
around the group centre and additionally averaged for the four groups shown. The result is given in Fig. 09. The decay in the space between for $\mathrm{R}>2 \mathrm{R}_{\text {group }}$ can also be roughly described by a $\mathrm{R}^{-2}$ law.

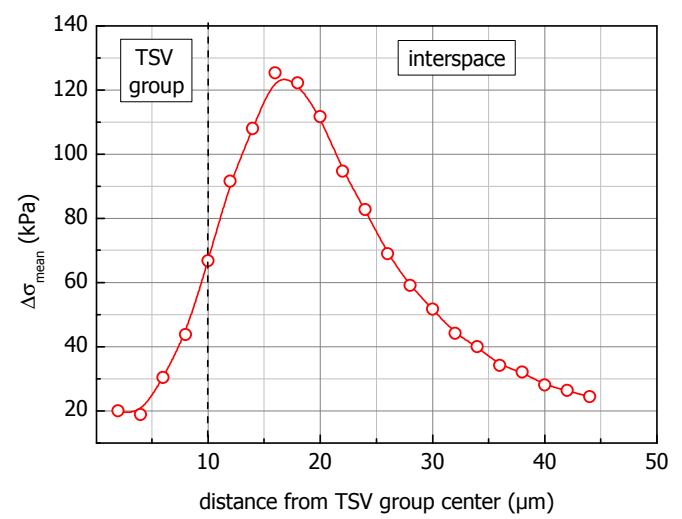

Fig. 09: Radial profile of 3-fold TSV after averaging in rings of $2 \mu \mathrm{m}$ width (see Fig. 08)

\subsubsection{5-fold TSV}

Fig. 10 presents the $\mathrm{G}$ maps of the 5-fold TSV matrix. In contrast to the examples discussed before this TSV design shows an opposite colour sequence of the butterflies. This means the anisotropy is caused by a compressive stress field. Furthermore, two butterflies (centre, left top corner) show a significantly increased shear stress magnitude.
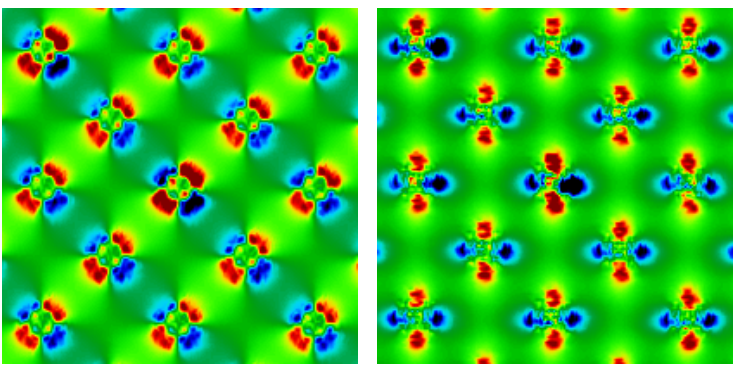

Fig. 10: Shear stress equivalent maps $(625 \mu \mathrm{m} \mathrm{x}$ $625 \mu \mathrm{m}$ ) of matrix of 5-fold TSV (length $100 \mu \mathrm{m}$, diameter $10 \mu \mathrm{m}$ ) using $0^{\circ}$ (left) and $45^{\circ}$ (right) polarization

Fig. 11 adds the corresponding map of $\Delta \sigma$. The white lines mark a dominant compressive stress field but there are tensile components along the diagonals in the space between the groups. Fig. 12 shows the averaged radial profiles determined as before. We observe a clear increase of magnitude for the both damaged TSV groups (left top and right bottom) in comparison to the inconspicuous ones (centre, left bottom and right top). The profiles in the space between for $\mathrm{R}>2 \mathrm{R}_{\text {group }}$ can be better fitted by an exponential decay than by a potential one.

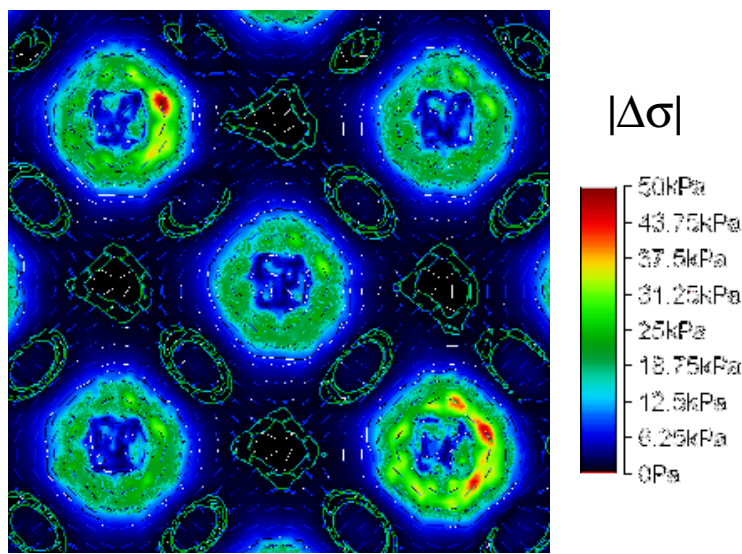

Fig. 11: Calculated $\Delta \sigma$ map $(400 \mu \mathrm{m} \times 400 \mu \mathrm{m})$ of 5fold TSV

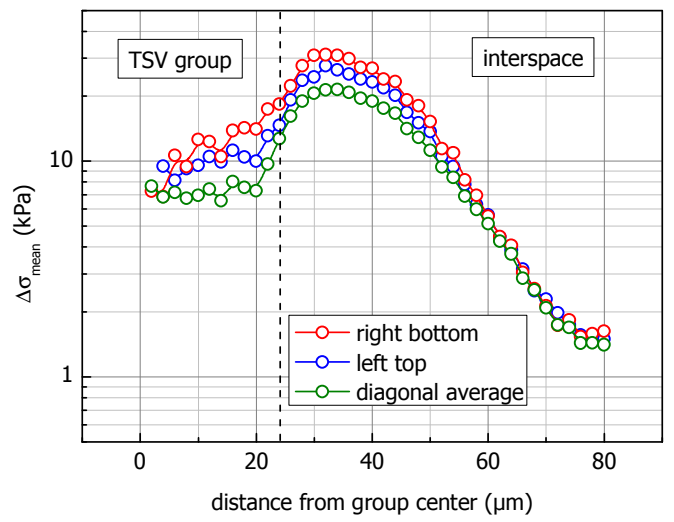

Fig. 12: Radial profiles of damaged and undamaged 5-fold TSV after averaging in rings of $2 \mu \mathrm{m}$ width (see Fig. 11) 


\section{Discussion}

The maps in Fig. 04, 06 and 08 show "butterflies" as known for radial-symmetric fields of point-like stress sources. However, the radial profiles determined from the converted $\Delta \sigma$ maps reveal that the "field" does not follow a simple $\mathrm{R}^{-2}$ law. The most significant difference observed is an increase of magnitude for $\mathrm{R}<$ $\mathrm{n} \times \mathrm{R}_{\text {group }}(\mathrm{n} \approx 10$ for single TSV, $\mathrm{n} \approx 2$ for 3 - and 5 -fold TSV). At the moment, the area $\mathrm{R} \approx \mathrm{R}_{\text {group }}$ should be taken out of discussion because the conditions of reflection are unclear yet. Blurring of profile by scanning with a Gaussian probe can not be excluded.

For the deviance from the Lamé law we see two main reasons. First, the stress anisotropy $\Delta \sigma$ should be treated as different from the single components of $\sigma$ in radial and azimuthal direction. Secondly, we have already noted that SIREX is an integrating probe. We assume a stress gradient in normal direction for one or both $\sigma$ components. In particular, it seems to be possible that the sign of $\Delta \sigma$ changes in depth. It is a fact that the stress near a TSV changes with depth, and that there is also a vertical component, also depending on depth, as found by Raman studies [7].

Finally, we note that the maximum of magnitude for the single and 3 -fold TSV is significantly higher than that for the more extended 5-fold TSV.

Consequently, a blind "hole" has to be discussed as a 3-dimensional problem. Modelling of the stress state in dependence on geometry, in particular on aspect ratio and pitch is the topic of current investigations.

\section{Summary}

We have studied Cu-filled TSV of different design and geometry by use of the photo-elastic microscope SIREX. The lateral distribution of the stress anisotropy represented by the difference of the in-plane principal stress components $\Delta \sigma$ is significantly different for single, 3-fold and 5-fold TSV. The dominant in-plane component of stress in the silicon matrix surrounding the studied single and 3-fold TSV is tensile, however, opposite to what is expected, compressive in the 5-fold TSV matrix. The averaged radial profiles of $\Delta \sigma$ show an increasing and a decreasing part. For the single and 3 -fold TSV the decay follows roughly a $\mathrm{R}^{-2}$ law, for the 5 -fold TSV rather an exponential one. In particular, maximum and decay are increased for damaged TSV groups.

We assume that even the raw "butterfly" can be used as fingerprint of defects what ensures a short time of inspection. A formal measure for the integrity of TSV has to be deduced. For this purpose, comprehensive studies of statistical relevance and complementary investigations by means of independent probes e. g. FIB (Focussed Ion Beam) are necessary.

\section{References}

[1] I. De Wolf, A. Khaled, M. Herms, M. Wagner, T. Djuric, P. Czurratis and S. Brand: Failure and Stress Analysis of Cu TSV using GHz-Scanning Acoustic Microscopy and Scanning Infrared Polariscopy; Conf. Proceed. ISTFA 2015, Portland, Oregon, USA, Nov. 2015, 119-125

[2] H. D. Geiler, H. Karge, M. Wagner, St. Eichler, M. Jurisch, U. Kretzer, and M. Scheffer-Czygan: Photoelastic Characterization of Residual Stress in GaAs-wafers; Materials Science in Semicond. Processing 9 (2006) 345-350

[3] D. Hull and D. J. Bacon: Introduction to Dislocations, $3^{\text {rd }}$ ed., Internat. Ser. On Materials Science and Technology, vol. 35, Pergamon Press (1984)

[4] H. D. Geiler, W. Kürner, and O. Storbeck: Photoelastic Imaging of Process Induced Defects in 300mm Silicon Wafers; Mat. Res. Soc. Symp. Proc. Vol. 591 (2000) 249-253

[5] W. Guo, G. Van der Plas, A. Ivankovic, V. Cherman, G. Eneman, B. De Wachter, M. Togo, A. Redolfi, S. Kubicek, Y. Civale, T. Chiarella, B. Vandevelde, K. Croes, I. De Wolf, I. Debusschere, A. Mercha, A. Thean, G. Beyer, B. Swinnen, and E. Beyne: Impact of Through Silicon Via Induced Mechanical Stress on Fully Depleted Bulk FinFET Technology; IEDM12 (2012) 431-433

[6] M. Herms, M. Wagner, A. Molchanov, P. Lin, I. De Wolf and M. Zhao: Materials Characterization and Device Analysis for Evaluation of Semiconductor Processes by Highly-sophisticated Photoelastic Stress Measurement Technique; Phys. Status Solidi C12 (2015) 1085-1089.

[7] I. De Wolf: Relation between Raman frequency and triaxial stress in $\mathrm{Si}$ for surface and cross sectional experiments in microelectronic components. J. Appl. Phys. 118, 053101 (2015) 